\title{
The human corpus callosum and the controversy about a sexual dimorphism
}

\author{
SERGE WEIS \\ Medical University of Lübeck, Lübeck, Federal Republic of Germany \\ GERMAIN WEBER \\ Ludwig Boltzmann Institute of Research on Brain-Damaged Children, Vienna, Austria \\ and \\ EMANUEL WENGER and MELITTA KIMBACHER \\ Austrian Academy of Sciences, Vienna, Austria
}

\begin{abstract}
The human corpus callosum was investigated with regard to sexual dimorphism by means of morphometry. At the macroscopical level, no part of the corpus callosum showed significant differences between the sexes. The concept that sex-related functional hemispherical discrepancies are correlated with the gross anatomy of the human corpus callosum was not confirmed.
\end{abstract}

De Lacoste-Utamsing and Holloway (1982) reported gender differences in the shape and cross-sectional area of the posterior part of the human corpus callosum. They described the female splenium to be more bulbous and larger than its male counterpart. They supposed that their findings, based on nine male and five female autopsy brains, could be related to possible gender differences in the degree of lateralization for visuospatial functions. In an extension and replication, Holloway and de Lacoste (1986), using eight brains of each sex, reproduced the same results. In addition, they reported that the total area of the corpus callosum of females was absolutely, as well as relatively, larger than that of males. Similar sex-related differences in the morphology of the callosal splenium have been reported in human fetuses (Baack, de LacosteUtamsing, \& Woodward, 1982; de Lacoste, Holloway, \& Woodward, 1986) and in other primates (de Lacoste \& Woodward, 1983). The results of de Lacoste-Utamsing and Holloway's (1982) study have already been introduced as facts by Kelly (1985) in a major neuroscience textbook.

Although Witelson (1985) found differences in the size of the corpus callosum between left- and right-handers, she could not demonstrate gender differences in the callosal splenium of her autopsy brains. Bell and Variend (1985) investigated a sample of 28 male and 16 female autopsy brains of white children aged from birth to 14 years. A one-way analysis of covariance, with age and brain weight as covariates, did not show differences in splenial width or area between males and females under 2 years of age. Furthermore, Weber and Weis (1986), using a larger sample of autopsied brains (18 brains of

This paper is dedicated to Herbert Haug on the occasion of his 68 th birthday. Correpondence may be addressed to Serge Weis, Institute of Anatomy, Medical University of Lübeck, Ratzeburger Allee 160, D-2400 Lübeck 1, Federal Republic of Germany. each sex), also failed to replicate de Lacoste's results. Although measurements of the maximal splenial width and the total length, cross-sectional area of the posterior third, fourth, and fifth of the corpus callosum were done, no significant differences between the sexes could be revealed. An additional form factor, evaluated for the splenium, also failed to show sex differences. The data of these parameters were processed by means of a discriminant analysis, with sex as a classification variable. However, only $58 \%$ of the cases were correctly classified. It was concluded that the size and shape of the splenium corporis callosi vary widely from one person to another, but not significantly between the sexes. These results recently have been confirmed by Demeter, Ringo, and Doty (1988).

Using nuclear magnetic resonance (NMR) imaging as a method to study the issues of gross-anatomical gender differences in the brain, it is possible to investigate a living population and to avoid the high average age of an autopsy sample, thus eliminating two major sources of artifacts in this research field.

Bleier, Houston, and Byne (1986) measured the corpus callosum on NMR images in 17 men and 22 women. Their results did not confirm gender-associated differences in splenial area or maximum splenial width; rather, they observed large variations in callosal size and shape among individuals irrespective of sex and age.

Oppenheim, Lee, Nass, and Gazzaniga (1987) calculated relative measurements for the corpus callosum of 40 men and 40 women. They did not find significant sexrelated differences for callosal areas, maximal callosal width, or callosal curvature. Kertesz, Polk, Howell, and Black (1987) reported on a magnetic resonance imaging study of the corpus callosum, using a large sample (52 subjects of each sex). They did not detect sex differences in callosal area ratios for the sagittal cross-sectional area, 
but found large individual variations in callosal size and shape. Recently, Byne, Bleier, and Houston (1988) reported on an NMR-imaging investigation of the corpus callosum carried out on 15 males and 22 females. Their study confirmed the previous observation that individual variability was more important than the variability between the sexes.

In the present paper, additional data on the corpus callosum, obtained by NMR imaging, is presented. The measurements were extended to include an evaluation of the total corpus callosum and some new parameters.

\section{METHOD}

The subjects were 20 males and 26 females aged $24-83$ years. The average ages of the male and female groups were 44.1 ( $S D$ $=17.1)$ and $48.9(S D=17.4)$ years, respectively. The subjects were selected from a sample that had undergone NMR imaging because of neurological disturbances, but whose neuroradiological reports were free of neuropathological signs. Thus, people with disease states involving the corpus callosum, such as multiple sclerosis (Huber et al., 1987) and demented states (Yoshii et al., 1986), and cases with brain tumors and hydrocephalus were not included in the subject sample. Since material was delivered by routine NMR imaging, no information on hand preference could be obtained.

Median-sagittal images were produced with the 0.5 Tesla superconductivity system GYROSCAN S5 (Phillips) using short TE/TR $(30 / 350)$ spin echo sequences, and then subjected to digital image analysis.

The outline of the corpus callosum was traced with the digitizer. The metric scale and the lowest points of the rostrum and the splenium were transmitted to the system.

The quantification was performed as illustrated in Figure $1 \mathrm{~A}$, in which $\mathrm{HI} 1$ = horizontal inferior 1 tangent to the most inferior point of the rostrum and the splenium; VA $1=$ vertical anterior 1 tangent to the most anterior point of the genu and right-angled to $\mathrm{HI} 1$; VP $1=$ vertical posterior 1 tangent to the most posterior point of the splenium and right-angled to $\mathrm{HI} 1$; HS $1=$ horizontal superior 1 tangent to the most superior point of the trunk, parallel to HI 1 and right-angled to VA 1 and VP 1.

The callosal height was defined as the distance between $\mathrm{HI} 1$ and HS 1; the callosal length was defined as the distance between VA 1 and VP 1 . The callosal length was subdivided into $2,3,4$, and 5 parts. The profile area of these different parts was evaluated consecutively (see Figure 1A).

To avoid the vague definition of the "maximal splenial width" given by de Lacoste-Utamsing and Holloway (1982) and to make the evaluation reproducible, we measured the width of the posterior fifth of the corpus callosum by calculating the maximal diameter of the splenium parallel to HI 1 , called MDX, and the maximal diameter of the splenium parallel to VP 1, called MDY.

The crossing point of MDX and MDY served as a reference point for the rotatory diameter measurement. Starting from MDY, at regular intervals of $10^{\circ}$, lines connecting the splenial outlines and passing through the crossing point were traced. The lengths of these 10 lines were measured. This procedure allowed reproducible measurements of the maximal diameters of the splenium (see Figure 1B).

\section{RESULTS}

The results of the measurements for the callosal height, length, and profile area, as well as for the profile areas of the different subdivisions of the callosal length in 2 ,
3 , 4, and 5 parts, are listed in Table 1 . No significant differences between the sexes could be found.

The results obtained by the rotatory diameter measurements are listed in Table 2. No significant differences could be detected between the male and female groups.

\section{DISCUSSION}

In the present study, sex differences could not be demonstrated in any part of the corpus callosum. None of the classical parameters (i.e., profile area and callosal length) nor rotatory diameter measurements were found to differ between males and females. These results are consistent with the morphometric measurements of Bleier et al. (1986), Oppenheim et al. (1987), Kertesz et al. (1987), and Byne et al. (1988), although the evaluation procedures differed in various ways between the research groups. The paper of Bleier et al. (1986) lacked information on the quantification procedure. It seems that one crucial point is how splenial width is defined. Although they presented results on splenial width, Byne et al. (1988) failed to indicate their measure of splenial width on the figures of the corpus callosum. Oppenheim et al. (1987) divided the corpus callosum into five parts and defined the splenial width in a manner similar to the present work. They did not, however, present absolute measurements of splenial width; rather, their measurement of callosal distance and area were presented as relative data (i.e., as a percentage of the total callosal area or distance). It can only be assumed that the images from which they made their measurements were lacking a metric scale. Finally, Kertesz et al. (1987) presented results on a crosssectional area of the corpus callosum without considering quantification data of its subdivided parts.

Recently, some functional differences in interhemispheric transfer have been reported. St. John, Shields, Krahn, and Timney (1987) assessed the reliability of estimates in interhemispheric transmission time derived from verbal and unimanual responses. They found that, for unimanual response conditions, interhemispheric transmission times for the female subjects were estimated to be longer and exhibited poorer reliabilities than for the male subjects. However, the verbal response conditions showed no differences in the mean duration of the estimates between females and males.

In another study, St. John and Timney (1986) examined the transmission of information through callosal fibers by simple unimanual reaction time in human strabismics. They observed that the estimates of cross-callosal transmission time for strabismics were distinctly longer than for the normal controls. St. John and Timney concluded that human strabismics had abnormalities in the callosal fibers that are involved in interhemispheric transmission of visual information.

Byne et al. (1988) argued that if variations in callosal size or shape are interpreted as parameters of cognitive functions, then such interpretations must be based on known correlations between callosal size or shape and par- 


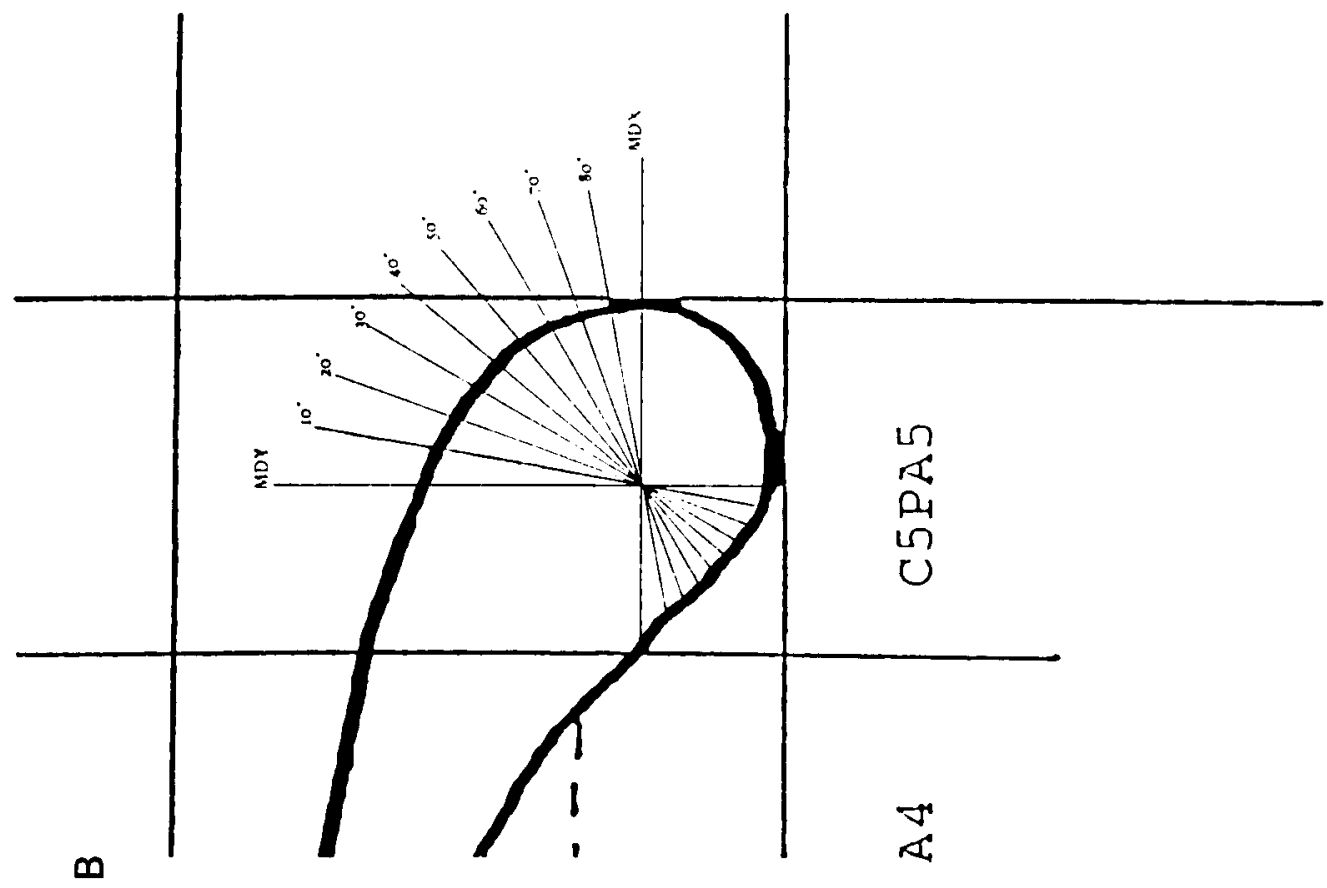

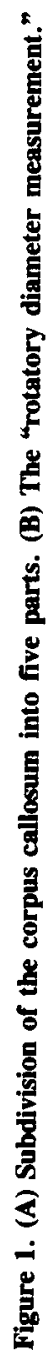

$\varangle$

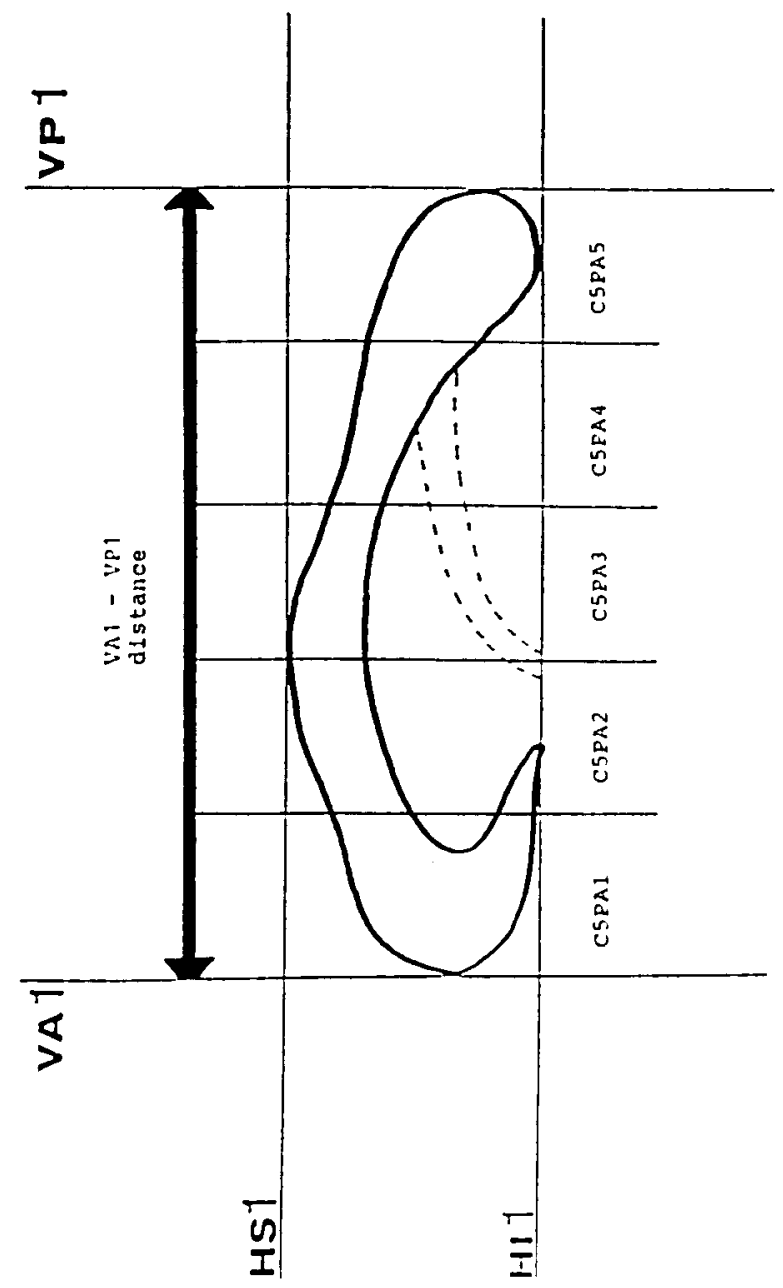


ticular cognitive functions. At present, there is no evidence for such correlations. Indeed, Kertesz et al. (1987) showed that callosal area did not correlate with brain size or with measures of lateralization for hand performance, dichotic listening, or visual field preference. They did not indicate, however, what quantification procedure was used to estimate brain size.

In spite of the fact that this investigation and others have not found reliable sex differences in the gross anatomy of the human corpus callosum, functional differences between the sexes in interhemispheric transfer have been described. Functional interhemispheric differences may result either in a changed electrophysiological transmission pattern or in variations of the diameters of callosal axons. Whether these possible changes in the microstructure of callosal fibers could provide a basis for theories of sex differences in cognitive functions must be considered in careful investigations, since to our present knowledge only $2 \%$ of cortical neurons have commissural axons (Creutzfeldt, 1983).

The existing scientific trend of trying to relate nonpathological behavioral differences to differences in gross anatomy appears to be premature and of debatable value.

Table 1

Mean Values $(M s)$ and Standard Deviations $(S D s)$ of the Evaluated Parameters Compared in Both Sex Groups

\begin{tabular}{lrrrrr}
\hline & \multicolumn{2}{c}{ Female Group } & & \multicolumn{2}{c}{ Male Group } \\
\cline { 2 - 3 } \cline { 5 - 6 } Parameters & \multicolumn{1}{c}{$M$} & $S D$ & & \multicolumn{1}{c}{$M$} & \multicolumn{1}{c}{$S D$} \\
\hline CH & 26.84 & 2.91 & 26.66 & 3.14 \\
CL & 76.72 & 6.51 & 74.61 & 5.41 \\
CPA & 665.20 & 96.30 & 669.92 & 122.49 \\
C2PA1 & 331.77 & 51.02 & 341.64 & 66.28 \\
C2PA2 & 333.43 & 51.92 & 327.98 & 62.47 \\
C3PA1 & 255.65 & 41.12 & 267.02 & 54.22 \\
C3PA2 & 148.07 & 23.19 & 144.84 & 26.82 \\
C3PA3 & 261.48 & 42.22 & 257.75 & 50.69 \\
C4PA1 & 216.62 & 33.86 & 224.07 & 44.03 \\
C4PA2 & 114.22 & 19.51 & 115.99 & 24.02 \\
C4PA3 & 107.15 & 18.76 & 107.81 & 20.89 \\
C4PA4 & 226.28 & 37.96 & 220.16 & 43.99 \\
C5PA1 & 185.15 & 26.87 & 187.95 & 33.96 \\
C5PA2 & 93.00 & 16.61 & 98.78 & 23.64 \\
C5PA3 & 89.32 & 14.69 & 86.05 & 14.91 \\
C5PA4 & 89.26 & 17.25 & 93.97 & 21.54 \\
C5PA5 & 199.95 & 34.52 & 191.54 & 38.06 \\
\hline
\end{tabular}

Note-CH and CL are expressed in millimeters; all other parameters are expressed in square millimeters. Using the Mann-Whitney $U$ test, no significant differences could be detected between the male and female groups. List of abbreviations for callosal parameters: $\mathrm{CH}$, callosal height; CL, callosal length; CPA, callosal profile area; C2PA1, profile area of the first callosal half; C2PA2, profile area of the second callosal half; C3PA1, profile area of the first callosal third; C3PA2, profile area of the second callosal third; C3PA3, profile area of the third callosal third; C4PA1, profile area of the first callosal fourth; C4PA2, profile area of the second callosal fourth; C4PA3, profile area of the third callosal fourth; C4PA4, profile area of the fourth callosal fourth; C5PA1, profile area of the first callosal fifth; C5PA2, profile area of the second callosal fifth; C5PA3, profile area of the third callosal fifth; C5PA4, profile area of the fourth callosal fifth; C5PA5, profile area of the fifth callosal fifth.
Table 2

Mean Values (Ms) and Standard Deviations (SDs) of the Splenial Parameters Obtained by the Rotatory Diameter Measurement

\begin{tabular}{cccccc} 
& \multicolumn{2}{c}{ Female Group } & & \multicolumn{2}{c}{ Male Group } \\
\cline { 2 - 5 } Parameters & $M$ & $S D$ & & $M$ & $S D$ \\
\hline MDY & 13.13 & 1.83 & 13.09 & 1.82 \\
$+10^{\circ}$ & 12.02 & 1.99 & 12.15 & 1.82 \\
$+20^{\circ}$ & 11.49 & 2.40 & 11.73 & 1.83 \\
$+30^{\circ}$ & 11.26 & 2.69 & 11.53 & 1.80 \\
$+40^{\circ}$ & 11.40 & 2.96 & 11.53 & 1.84 \\
$+50^{\circ}$ & 11.71 & 3.12 & 11.75 & 1.82 \\
$+60^{\circ}$ & 12.30 & 3.30 & 12.44 & 1.70 \\
$+70^{\circ}$ & 13.36 & 3.33 & 13.61 & 1.60 \\
$+80^{\circ}$ & 14.98 & 3.42 & 15.07 & 1.79 \\
MSX & 17.78 & 2.41 & 17.26 & 2.65 \\
\hline
\end{tabular}

Note-All splenial diameters are expressed in millimeters. Using the Mann-Whitney $U$ test, no significant differences could be detected between the male and female groups. MDY $=$ the maximal diameter of the splenium parallel to vertical posterior $1 . \mathrm{MDX}=$ the maximal diameter of the splenium parallel to horizontal interior 1 .

Although many researchers obtain similar findings, there remain differences in the way the neuroanatomical parameters are measured. Before any definitive conclusions can be reached, it will be necessary to clearly define the quantification procedure used. We adhere to the conviction that the functional neuroanatomical issue can only be approached seriously after the major methodological issues are resolved.

\section{REFERENCES}

BaAck, J., De Lacoste-Utamsing, M. C., \& Woodward, D. J. (1982). Sexual dimorphism in the human fetal corpora callosa. Neuroscience Abstracts, 8, 18.

BeLl, A. D., VARIEND, S. (1985). Failure to demonstrate sexual dimorphism of the corpus callosum in childhood. Journal of Anatomy, 143, 143-147.

Bleier, R., Houston, L., BYNE, W. (1986). Can the corpus callosum predict gender, age, handedness, or cognitive differences? Trends in Neurosciences, 9, 391-394.

Byne, W., Bleier, R., \& Houston, L. (1988). Variations in human corpus callosum do not predict gender: A study using magnetic resonance imaging. Behavioral Neuroscience, 102, 222-227.

Creutzfeldt, O. D. (1983). Cortex cerebri. Berlin: SpringerVerlag.

DE Lacoste, M. C., \& Woodward, D. J. (1983). Neocortical commissural size and sex differences in primate brain. Neuroscience Abstracts, 9, 45

de Lacoste, M. C., Holloway, R. L., \& Woodward, D. J. (1986). Sex differences in the fetal human corpus callosum. Human Neurobiology, 5, 93-96.

de Lacoste-Utamsing, M. C., \& Holloway, R. L. (1982). Sexual dimorphism in the human corpus callosum. Science, 216, 1431-1432.

Demeter, S., Ringo, J. L., \& Doty, R. W. (1988). Morphometric analysis of the human corpus callosum and anterior commissure. $\mathrm{Hu}$ man Neurobiology, 6, 219-226.

Holloway, R. L., \& DE Lacoste, M. C. (1986). Sexual dimorphism in the human corpus callosum: An extension and replication study. Human Neurobiology, 5, 87-91.

Huber, S. J., Paulson, G. L., Shutrleworth, E. C., Chakeres, D., Clapp, L. E., Pakalnis, A., Weiss, K., \& Rammohan, K. (1987). Magnetic resonance imaging correlates of dementia in multiple sclerosis. Archives of Neurology, 44, 732-736. 
KeLLY, D. D. (1985). Sexual differences of the nervous system. In E. R. Kandel \& G. M. Schwartz (Eds.), Principles of neural science (2nd ed.). Amsterdam: Elsevier.

Kertesz, A., Polk, M., Howell, J., \& Black, S. E. (1987). Cerebral dominance, sex and callosal size in MRI. Neurology, 37, 1385-1388.

Oppenheim, J. S., Lee, B. C. P., Nass, R., \&azzaniga, M. S. (1987). No sex-related differences in human corpus callosum based on magnetic resonance imagery. Annals of Neurology, 21, 604-606.

St. John, R., Shields, C., Krahn, P., \& Timney, B. (1987). The reliability of estimates of interhemispheric transmission times derived from unimanual and verbal response latencies. Human Neurobiology, 6, 195-202.

ST. JoHN, R., \& TIMNEY, B. (1986). Interhemispheric transmission delays in human strabismics. Human Neurobiology, 5, 97-103.
WEBER, G., \& WEIS, S. (1986). Morphometric analysis of the human corpus callosum fails to reveal sex-related differences. Journal fur Himforschung, 27, 237-240.

Witelson, S. F. (1985). The brain connection: The corpus callosum is larger in left-handers. Science, 229, 665-668.

Yoshil, F., Barker, W., Apicella, A., Chang, J., Sheldon, J., \& DuARA, R. (1986). Measurements of the corpus callosum (cc) on magnetic resonance (MR) scans: Effects of age, sex, handedness and disease. Neurology, 36(Suppl. 1), 133.

(Manuscript received March 2, 1988.) 